\title{
Drug approval regulation: the global impacts
}

\begin{abstract}
People want prescription drugs to be efficient as well to assist them in treating their ailments. The need of drug regulations was generated in the twentieth-century as a result of disasters, tragedies, and misfortunes with public health in the past. The legal regulatory document in the form of pharmacopeia came in existence to regulate quality and standard of the medical supplements given to patients. Today, the drug approval criteria in different pharmacopeias differ regionally. Because of the approval process variations, pharmaceutical businesses are facing difficulty in approving new pharmaceutical products for marketing in many countries simultaneously. The review contains regulatory requirements and also, economic and social impacts of variation on pharmaceutical businesses. An effort is made to find solutions by making regulatory requirements globalized.
\end{abstract}

Keywords: regulations, global, approval, drug, impact
Volume 4 Issue 6 - 2017

\author{
Jayshree D Patel, Gregorio Kahn \\ National University, USA
}

Correspondence: Jayshree D Patel, Scientist. IriSys, LLC, National University, San Diego, California, USA,

Email jdpatels@gmail.com

Received: May 12, 2017 | Published: May 23, 2017
Abbreviations: GLP, good laboratory practice; GCP, good clinical practice; cGMP, current good manufacturing practice; FDA, food and drug administration; EMA, european medicines agency; CDSCO, central drug standard control organization; MAA, marketing authorization application

\section{Introduction}

People want prescription drugs to be efficient as well as effective when treating their ailments. The public regulatory authorities are responsible for ensuring that pharmaceutical companies comply with regulations. It is legislation that requires drugs to be developed, tested, trialed, and manufactured in accordance with guidance so that they protect patients' well-being. Globally, regulatory authorities perform the watchdog role to ensure that animal studies comply with Good Laboratory Practice (GLP), clinical trials performed in accordance with Good Clinical Practice (GCP), and drug manufacturing companies follow current Good Manufacturing Practice (cGMP). All these good practices are compulsory requirements for all the drug developmental companies whom fulfill approval from regulatory agencies.

Currently, pharmaceuticals are considered one of the most highly regulated industries worldwide. Drug regulation is the control of drug use by regulatory authorities such as the US Food and Drug Administration (FDA), the European Medicines Agency (EMA), Central Drug Standard Control Organization (CDSCO) etc. The regulatory body ensures compliances in legal and regulatory aspects of the drug. Every country has its own regulatory authority, which is responsible for enforcing the rules and regulations. It issues the guideline to regulate drug development process, licensing, registration, manufacturing, marketing, and labeling of pharmaceutical products. Therefore, drug approval regulations differ globally. For the Marketing Authorization Application (MAA), a single regulatory approach to get permission in multiple countries can became a difficult task.

Every regulatory authority requires proper documentation before granting permission for a new drug. The requirement includes substantial evidence of safety, statistically significant data to demonstrate efficacy, and controlled Good Manufacturing Practice (cGMP) of the drug discovery stage, drug developability stage, pre-clinical stage, clinical drug development stage, manufacturing facility with appropriate quality and quantity of final therapeutic product. ${ }^{1}$ Expert teams with research and management responsibilities include reviewing research results, integrating new research results, planning research study, preparing a detailed drug development plan, monitoring the status of the research study, the development status and timelines, market survey, and reporting the progress status to management, works together to compete the task successfully. The role of pharmacopeia is to monitor the manufacturing stage that requires adherence to follow standard protocols to maintain quality standard drug for public consumptions.

\section{Global impact of regulation}

Approval and prescription criteria for some medicines vary in different countries. Scholars have reported that some medicines passed from European pharmacopeia did not stay competent with the United States pharmacopeia. The dissimilarities in the approval process is hindrance for the drug developers to sell their product in global market. The BRIC countries, Indonesia, Mexico, and Turkey are expected to account for approximately $20 \%$ of total pharmaceutical market by $2020 .^{2}$ The marketing approval criteria of MAA for new drug are different in every country. The variations in quality control parameters and guidelines have promoted illegal drug supplies in many developed countries. According to Drug Trafficking, ${ }^{3}$ every year an estimated \$1.6billion worth of medicine is smuggled from Asia to South East Asia and Africa. The United States is the most worthwhile market for international drug traffickers. Tons of illicit drug trafficking have been taken place in the Americas during first decade of twenty-first century. ${ }^{4}$ It is hard to get real data but estimated loss from international drug trafficking were nearing \$10trillion dollars annually. It is illegal therefore it is tax free and a multitrillion dollar international business. Raising awareness among citizens will reduce illegal entries and illicit drug consumptions.

In the $21^{\text {st }}$ century, the chance of diseases spreading from one country to another has increased drastically. SARS, avian influenza, Ebola, and Zica virus spread quickly across national borders and make it necessary to find a faster regulatory process for global public health security. The regulatory requirements of different countries vary from one another. Therefore, it is challenging for pharmaceutical companies to develop pharmaceutical products which can be 
simultaneously submitted in every country for approval. ${ }^{5}$ Some efforts were undertaken in 2009 by the Obama administration and his Drug Czar Gil Kerlikowske to redress this long-standing imbalance in U.S. drug policy, although the programs remain woefully underfunded. ${ }^{4}$ Globalization in pharmacopeia regulations will decrease the chance of marketing fake medicines. Drug approval processes need less tightened regulations, for example, if any medicine is in use for the human beings for more than five years in one country, the medicine should be approved for distribution in other countries.

\section{Conclusion}

Globalized criteria will bring harmonization among all regulations Pharmacopeia burdened regulations on the pharmaceutical companies. Quality standard is necessary to maintain public health worldwide. The strictness in regulations will raise the problem of drug smuggling in many developed countries. The illegal pharmaceutical products brings economic burden in countries' government and indirectly on GDP growth. Counterfeits medicines, privatization of regulations, and price variation will consider as negative impacts of globalization.

In the future, new technology and new products will create new regulatory challenges. Currently, the regulatory process is complicated and lengthy. The cost of full regulatory assessment is increasing and could become less affordable in terms of financial and human resources. Therefore, international, regional, and sub-reginal harmonization between regulatory authorities is necessary to avoid duplications in reports, costs, and time for regulatory review. This will translate to patients receiving access to new drugs more speedily and at lower costs; thus, will decrease the chances of illegal drug entry throughout boarders and patients will receive the proper medication in a timely manner.

\section{Acknowledgements}

None.

\section{Conflict of interest}

The author declares no conflict of interest.

\section{References}

1. Patel JD, Fadaei R. Cost of antibiotics in the society and economic approach. IOSR Journal of Economics and Finance. 2016;7(6):17-20.

2. Melgar H, Schwieterman L. Approval Process for Allopathic, Biological and Biotechnological Medications in Mexico. Regulatory Focus. 2009:33-38.

3. Drug Trafficking. Drug Trafficking: Immigration to the Unites States. 2015.

4. Bagley B. Drug trafficking and organized crime in the Americas. USA: Wooddrow Wilson International Center for Scholars; 2012.

5. Handoo S, Arora V, Khera D, et al. A comprehensive study on regulatory requirements for development and filling of generic drugs globally. Int $J$ Pharm Investig. 2012;2(3):99-105. 